\title{
The basics of epithelial-mesenchymal transition
}

\author{
Raghu Kalluri',2 and Robert A. Weinberg ${ }^{3}$ \\ 1Division of Matrix Biology, Beth Israel Deaconess Medical Center, and Department of Biological Chemistry and Molecular Pharmacology, \\ Harvard Medical School, Boston, Massachusetts, USA. ${ }^{2 H a r v a r d-M I T ~ D i v i s i o n ~ o f ~ H e a l t h ~ S c i e n c e s ~ a n d ~ T e c h n o l o g y, ~ B o s t o n, ~ M a s s a c h u s e t t s, ~ U S A . ~}$ \\ ${ }^{3}$ Whitehead Institute for Biomedical Research, Ludwig Center for Molecular Oncology, and Department of Biology, \\ Massachusetts Institute of Technology, Cambridge, Massachusetts, USA.
}

\begin{abstract}
The origins of the mesenchymal cells participating in tissue repair and pathological processes, notably tissue fibrosis, tumor invasiveness, and metastasis, are poorly understood. However, emerging evidence suggests that epithelial-mesenchymal transitions (EMTs) represent one important source of these cells. As we discuss here, processes similar to the EMTs associated with embryo implantation, embryogenesis, and organ development are appropriated and subverted by chronically inflamed tissues and neoplasias. The identification of the signaling pathways that lead to activation of EMT programs during these disease processes is providing new insights into the plasticity of cellular phenotypes and possible therapeutic interventions.
\end{abstract}

\section{What is epithelial-mesenchymal transition?}

An epithelial-mesenchymal transition (EMT) is a biologic process that allows a polarized epithelial cell, which normally interacts with basement membrane via its basal surface, to undergo multiple biochemical changes that enable it to assume a mesenchymal cell phenotype, which includes enhanced migratory capacity, invasiveness, elevated resistance to apoptosis, and greatly increased production of ECM components (1). The completion of an EMT is signaled by the degradation of underlying basement membrane and the formation of a mesenchymal cell that can migrate away from the epithelial layer in which it originated.

A number of distinct molecular processes are engaged in order to initiate an EMT and enable it to reach completion. These include activation of transcription factors, expression of specific cell-surface proteins, reorganization and expression of cytoskeletal proteins, production of ECM-degrading enzymes, and changes in the expression of specific microRNAs. In many cases, the involved factors are also used as biomarkers to demonstrate the passage of a cell through an EMT (Figure 1).

The pioneering work of Elizabeth Hay first described an "epithelialmesenchymal transformation" using a model of chick primitive streak formation (2). In the intervening time, the term "transformation" has been replaced with "transition," reflecting in part the reversibility of the process and the fact that it is distinct from neoplastic transformation (1). The phenotypic plasticity afforded by an EMT is revealed by the occurrence of the reverse process - a mesenchymal-epithelial transition (MET), which involves the conversion of mesenchymal cells to epithelial derivatives. Relatively little is known about this process; the best-studied example is the MET associated with kidney formation, which is driven by genes such as paired box 2 (Pax2), bone morphogenetic protein 7 (Bmp7), and Wilms tumor 1 (Wt1) (3-5).

\section{Why does EMT occur?}

The concept of cell division as a way to generate more cells and expand tissue size emerged about 150 years ago (6). Central to

Conflict of interest: The authors have declared that no conflict of interest exists. Nonstandard abbreviations used: BMP, bone morphogenetic protein; EMT, epithelial-mesenchymal transition; EndMT, endothelial-mesenchymal transition; FSP1, fibroblast-specific protein 1; LEF, lymphoid enhancer binding factor; MET, mesenchymal-epithelial transition.

Citation for this article: J. Clin. Invest. 119:1420-1428 (2009). doi:10.1172/JCI39104. this concept was an understanding that all cells in a body derive from other cells and the resulting deduction that ultimately all are derived from a single cell, the fertilized egg. An additional level of complexity came from the realization that cells can assume various phenotypic states during development, that is, they can undergo the process of differentiation. As was learned more recently, during specific steps of embryogenesis and organ development, the cells within certain epithelia appear to be plastic and thus able to move back and forth between epithelial and mesenchymal states via the processes of EMT and MET (7). Upon completion of the development of epithelial tissues, the epithelial cells typically exert tissue-specific function, while the mesenchymal cells in such tissue play a supporting role. Implied in this notion was the further idea that a state of terminal differentiation is necessary to carry out such specialized functions and that cells are maintained in a permanent state of differentiation once development is complete.

This concept has been challenged by numerous observations that the cells within a terminally differentiated epithelium can indeed change their phenotype through activation of an EMT program, which enables transdifferentiation, resulting in the conversion of epithelial cells to mesenchymal derivatives during development and adulthood. These programs can also be activated in association with tissue repair and pathological stresses, including those creating various types of inflammation and high-grade carcinomas. Accordingly, EMTs now constitute recognized mechanisms for dispersing cells in embryos, forming mesenchymal cells in injured tissues, and initiating the invasive and metastatic behavior of epithelial cancers.

\section{Classification of EMT into three different subtypes}

EMTs are encountered in three distinct biological settings that carry very different functional consequences (Figure 2). While the specific signals that delineate the EMTs in the three discrete settings are not yet clear, it is now well accepted that functional distinctions are apparent. A proposal to classify EMTs into three different biological subtypes based on the biological context in which they occur was discussed at a 2007 meeting on EMT in Poland and a subsequent meeting in March 2008 at Cold Spring Harbor Laboratories. The EMTs that are associated with implantation, embryo formation, and organ development are organized to generate diverse cell types that share common mesenchymal phenotypes. This class of EMTs, which we and others (8) propose to term 


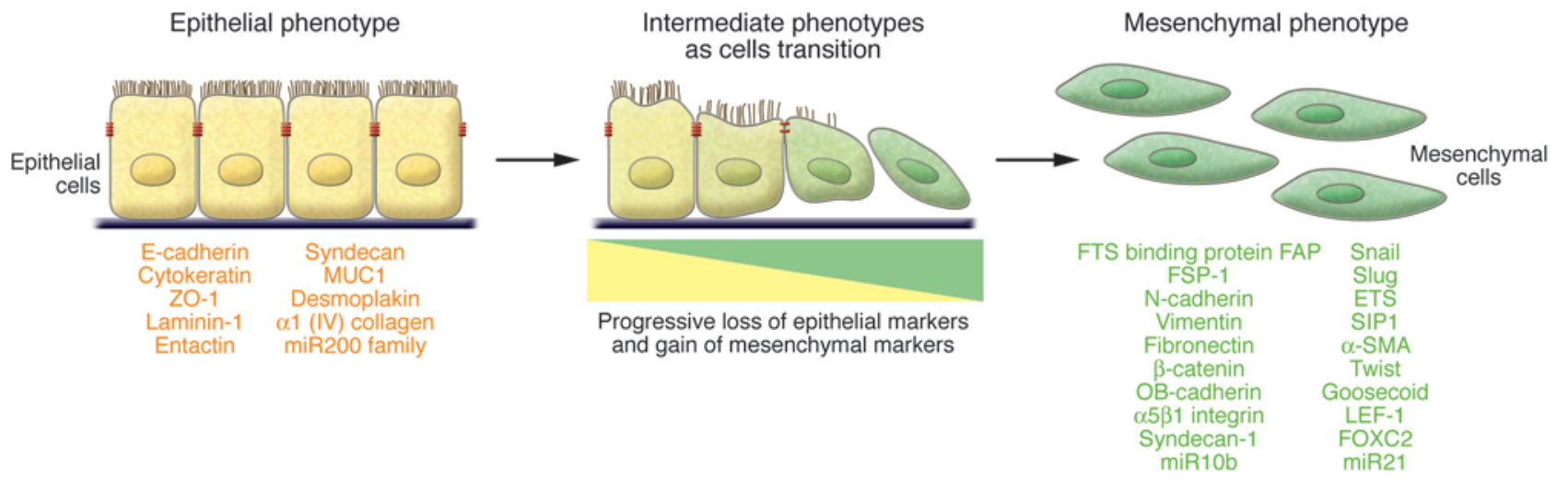

Figure 1

EMT. An EMT involves a functional transition of polarized epithelial cells into mobile and ECM component-secreting mesenchymal cells. The epithelial and mesenchymal cell markers commonly used by EMT researchers are listed. Colocalization of these two sets of distinct markers defines an intermediate phenotype of EMT, indicating cells that have passed only partly through an EMT. Detection of cells expressing both sets of markers makes it impossible to identify all mesenchymal cells that originate from the epithelia via EMT, as many mesenchymal cells likely shed all epithelial markers once a transition is completed. For this reason, most studies in mice use irreversible epithelial cell-lineage tagging to address the full range of EMT-induced changes. ZO-1, zona occludens 1; MUC1, mucin 1, cell surface associated; miR200, microRNA 200; SIP1, survival of motor neuron protein interacting protein 1; FOXC2, forkhead box C2.

"type 1," neither causes fibrosis nor induces an invasive phenotype resulting in systemic spread via the circulation. Among other outcomes, these type 1 EMTs can generate mesenchymal cells (primary mesenchyme) that have the potential to subsequently undergo a MET to generate secondary epithelia.

The EMTs associated with wound healing, tissue regeneration, and organ fibrosis are of a second type. In these type 2 EMTs, the program begins as part of a repair-associated event that normally generates fibroblasts and other related cells in order to reconstruct tissues following trauma and inflammatory injury. However, in contrast to type 1 EMTs, these type 2 EMTs are associated with inflammation and cease once inflammation is attenuated, as is seen during wound healing and tissue regeneration. In the setting of organ fibrosis, type 2 EMTs can continue to respond to ongoing inflammation, leading eventually to organ destruction. Tissue fibrosis is in essence an unabated form of wound healing due to persistent inflammation.

Type 3 EMTs occur in neoplastic cells that have previously undergone genetic and epigenetic changes, specifically in genes that favor clonal outgrowth and the development of localized tumors. These changes, notably affecting oncogenes and tumor suppressor genes, conspire with the EMT regulatory circuitry to produce outcomes far different from those observed in the other two types of EMT. Carcinoma cells undergoing a type 3 EMT may invade and metastasize and thereby generate the final, life-threatening manifestations of cancer progression. Importantly, cancer cells may pass through EMTs to differing extents, with some cells retaining many epithelial traits while acquiring some mesenchymal ones and other cells shedding all vestiges of their epithelial origin and becoming fully mesenchymal. It is still unclear what specific signals induce type 3 EMTs in carcinoma cells. For example, such signals may originate in the tumor stroma that is associated with many primary carcinomas.

While these three classes of EMTs represent distinct biological processes, a common set of genetic and biochemical elements appears to underlie and thus enable these outwardly diverse phenotypic programs. As more experiments are performed in the future, we will learn more regarding the similarities and differences among the three classes of EMTs.

\section{Type 1 EMT: EMT during implantation, embryogenesis, and organ development}

Following the earliest stages of embryogenesis, the implantation of the embryo and the initiation of placenta formation are both associated with an EMT that involves the parietal endoderm (9). In particular, the trophoectoderm cells, which are precursors of the cytotrophoblast, undergo an EMT in order to facilitate invasion of the endometrium and the subsequent proper anchoring of the placenta, enabling its function in nutrient and gas exchange (10, 11). As described briefly below, this is only the first of many type 1 EMTs that accompany and underlie embryonic morphogenesis; more detail regarding some of the EMTs that occur during development are discussed elsewhere (12).

A fertilized egg undergoes gastrulation, generating three germ layers. Initially, a primitive streak is generated in the epiblast layer (13). The epithelial cells in this tissue express E-cadherin and exhibit apical-basal polarity. Formation of the primitive streak is considered to be the first sign of gastrulation, which leads in turn to the formation of the three germ layers that generate all tissue types of the body. The primitive streak is formed from a furrowed invagination in the midline of the epiblast layer that forms initially at the lower extremity of the embryo and, later on, extends in the direction of the future head. The epithelial-like cells of the epiblast undergo programmed changes dictated by specific expression of proteins associated with cell migration and differentiation (14).

Once formed, the primitive streak, acting via invagination or ingression (depending on the species studied), generates the mesendoderm, which subsequently separates to form the mesoderm and the endoderm via an EMT (also known as epiblast-mesoderm transition) (2) by replacing the hypoblast cells, which presumably either undergo apoptosis or contribute to the mesoderm layer via an EMT. The embryonic mesoderm that forms between the epiblast and hypoblast eventually gives rise to primary mesenchyme associated with the axial, paraxial, intermediate, and lateral 


\section{A Type 1 EMT}
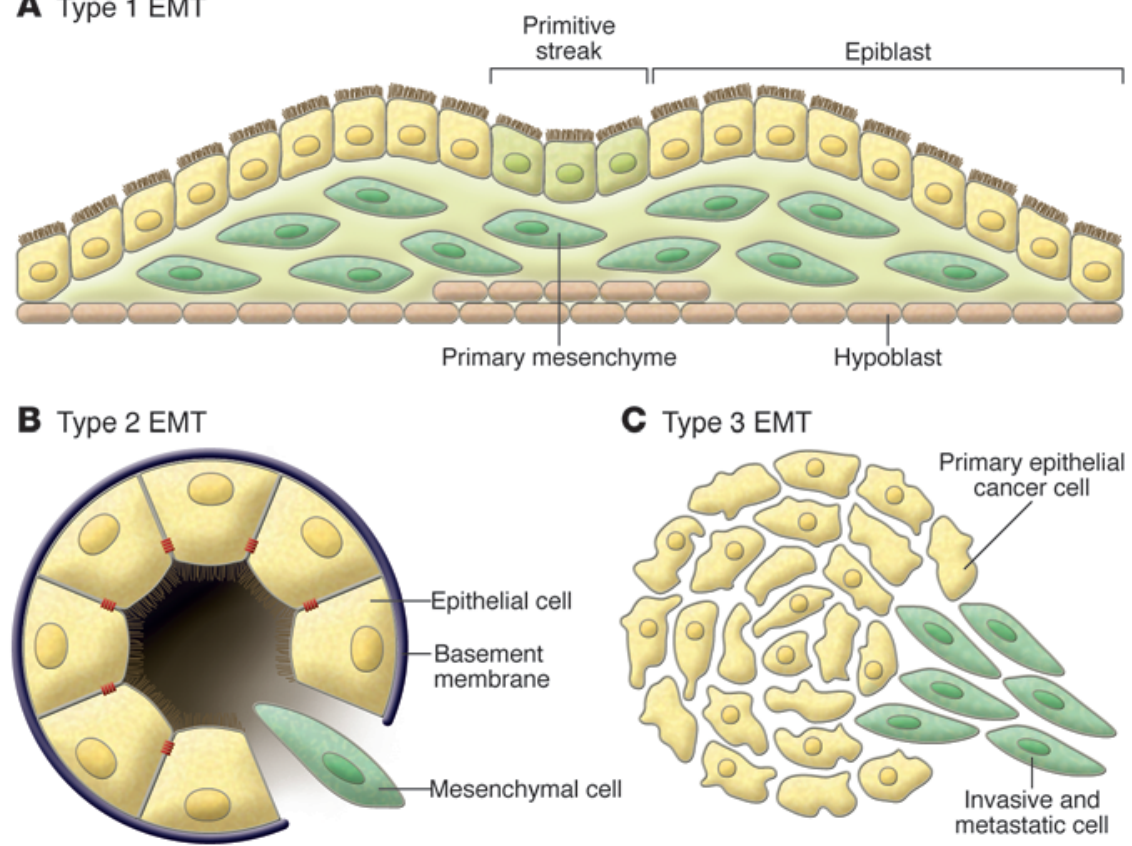

C Type 3 EMT

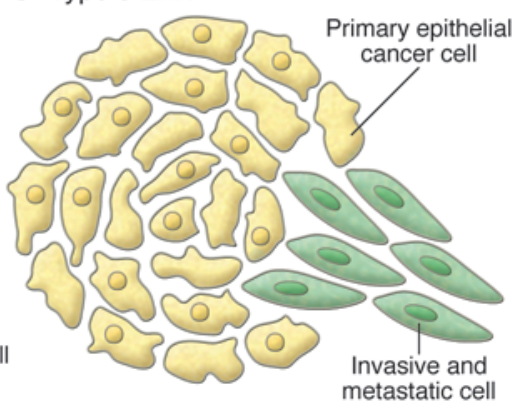

\section{Figure 2}

Different types of EMT. (A) Type 1 EMT is associated with implantation and embryonic gastrulation and gives rise to the mesoderm and endoderm and to mobile neural crest cells. The primitive epithelium, specifically the epiblast, gives rise to primary mesenchyme via an EMT. This primary mesenchyme can be re-induced to form secondary epithelia by a MET. It is speculated that such secondary epithelia may further differentiate to form other types of epithelial tissues and undergo subsequent EMT to generate the cells of connective tissue, including astrocytes, adipocytes, chondrocytes, osteoblasts, and muscle cells. (B) EMTs are re-engaged in the context of inflammation and fibrosis and represent the type 2 EMTs. Unlike the type 1 EMT, the type 2 EMT is expressed over extended periods of time and can eventually destroy an affected organ if the primary inflammatory insult is not removed or attenuated. (C) Finally, the secondary epithelia associated with many organs can transform into cancer cells that later undergo the EMTs that enable invasion and metastasis, thereby representing type 3 EMTs. plate mesodermal layers (15). Cells of the primary mesenchyme exhibit enhanced migratory properties when compared with those of the epiblast and the hypoblast (15).

At the biochemical level, the EMT associated with gastrulation is dependent on and orchestrated by canonical Wnt signaling, and embryos deficient in Wnt3 cannot undergo the EMT associated with gastrulation $(16,17)$. The subsequent formation of the primitive streak is associated with expression of Wnt8c, and ectopic expression of Wnt8c in embryos leads to multiple primitive streaks $(18,19)$. TGF- $\beta$ superfamily proteins, notably Nodal and Vg1, mediate the action of Wnts, and their deficiencies can lead to mesodermal defects due to the absence of functional EMTs (20-24). Wnts also cooperate with FGF receptors to help regulate an EMT associated with gastrulation (25-28). The Snail, Eomes, and Mesps transcription factors orchestrate the EMT associated with gastrulation (29-31). For example, Snail represses E-cadherin and induces EMT mediated by cell adhesion molecules, such as occludins and claudins, and by polarity genes, such as Discs large $(D l g)$ and Crumbs homolog 3 (Crb3) (32-34).

During embryonic development, an EMT involving the epithelial cells of the neuroectoderm gives rise to migratory neural crest cells (35). Initially, the premigratory neural crest cells express genes such as Sox, Snail, Slug, and forkhead box D3 (FoxD3), and these cells subsequently undergo an $\operatorname{EMT}(36,37)$. As a consequence, they then dissociate from the neural folds, become motile, and disperse to the different parts of the embryo, where they undergo further differentiation into, among other cell types, the melanocytes that provide pigment to the skin.

This EMT occurring in the neural crest is triggered by signaling pathways similar to those orchestrating the EMT associated with gastrulation. Thus, signaling pathways mediated by Wnts, FGFs, BMPs, c-Myb, and msh homeobox 1 (Msx-1) conspire to induce EMT (38-40). Among them, BMP most prominently induces the migratory property of neural crest cells. Noggin, an inhibitor of BMP, is essential in negatively regulating this activity, and its expression places a hold on EMT $(41,42)$. Additionally, E-cadherin and $\mathrm{N}$-cadherin, two cell adhesion molecules, need to be repressed in order for neural crest EMT to occur (43). By some estimates, EMT programs are deployed during several subsequent phases of embryogenesis, indicating the continued involvement of these programs later in development, such as the endothelial-mesenchymal transition (EndMT) that takes place during heart valve formation.

\section{Type 2 EMT: EMT associated with tissue regeneration and organ fibrosis}

Organ fibrosis, which occurs in a number of epithelial tissues, is mediated by inflammatory cells and fibroblasts that release a variety of inflammatory signals as well as components of a complex ECM that includes collagens, laminins, elastin, and tenacins (Figure 3). More specifically, such EMTs are found to be associated with fibrosis occurring in kidney, liver, lung, and intestine (44-47). Some of the earliest proof of this came from the study of transgenic mice bearing germ-line reporter genes whose expression was driven by epithelial cell-specific promoters. The behavior of these reporters provided direct evidence for epithelial cells serving, via EMTs, as important precursors of the fibroblasts that arise during the course of organ fibrosis (48-50).

Fibroblast-specific protein 1 (FSP1; also known as S100A4 and MTS-1), an S100 class of cytoskeletal protein, $\alpha$-SMA, and collagen I have provided reliable markers to characterize the mesenchymal products generated by the EMTs that occur during the development of fibrosis in various organs $(48,49,51)$. These markers, along with discoidin domain receptor tyrosine kinase 2 (DDR2), vimentin, and desmin, have been used to identify epithelial cells of the kidney, liver, lung, and intestine that are in the midst of undergoing an EMT associated with chronic inflammation. Such cells continued to exhibit epithelial-specific morphology and molecular markers, such as cytokeratin and E-cadherin, but showed concomitant expression of the FSP1 mesenchymal marker and $\alpha$-SMA. Such cells are likely to represent the intermediate stages 


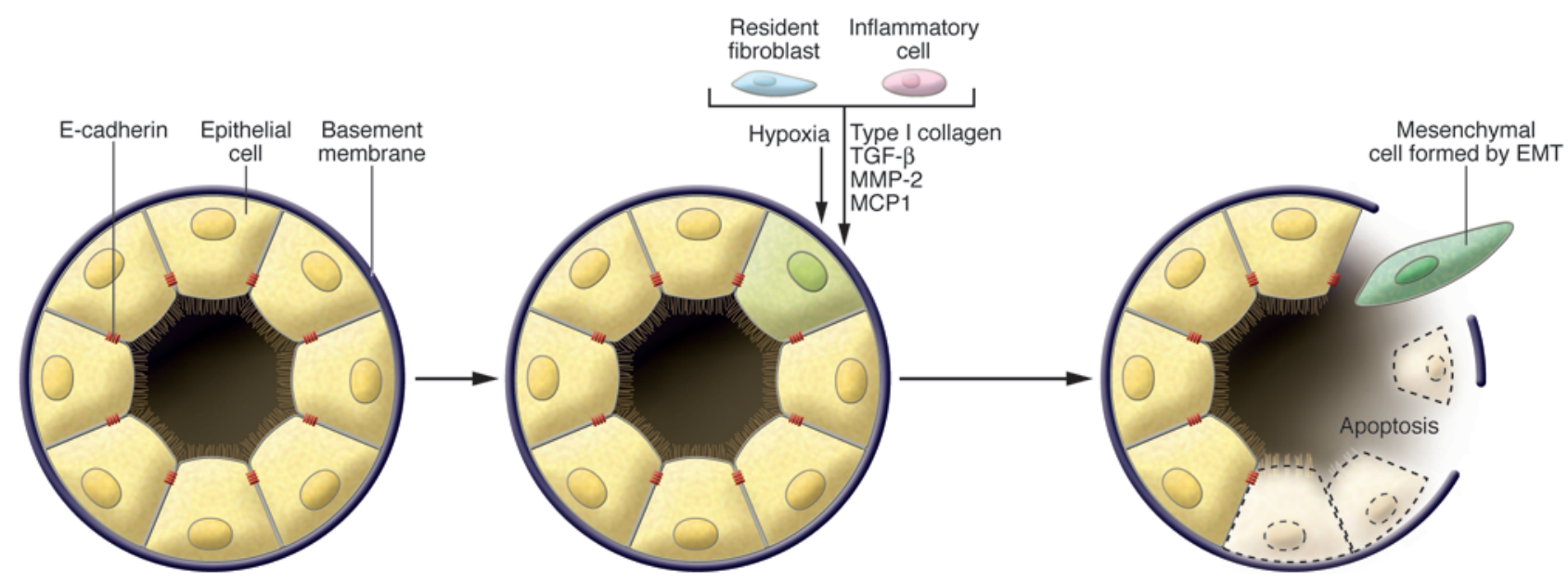

Figure 3

EMT and fibrosis. The EMTs associated with fibrosis are associated with inflammation and the generation of numerous types of molecules by inflammatory cells and resident activated fibroblasts (myofibroblasts). These molecules cause disruption of the epithelial layers via degradation of the basement membrane. The epithelial cells lose polarity and either undergo apoptosis (the majority of cells) or EMT (the minority of cells). MCP1, monocyte chemoattractant protein 1.

of EMT, when epithelial markers continue to be expressed but new mesenchymal markers have already been acquired. The behavior of these cells provided one of the first indications that epithelial cells under inflammatory stresses can advance to various extents through an EMT, creating the notion of "partial EMTs" (Figure 1). Eventually these cells leave the epithelial layer, negotiate their way through the underlying basement membrane, and accumulate in the interstitium of the tissue (52), where they ultimately shed all of their epithelial markers and gain a fully fibroblastic phenotype.

Recent experiments in mice have demonstrated that endothelial cells associated with the microvasculature can also contribute to the formation of mesenchymal cells during the course of fibrosis, doing so via an analogous process known as EndMT (44). This pathologic process echoes a similar normal process occurring during development (45). Thus, during embryogenesis, an EndMT occurs during the organization of the endocardial cushion and the heart valves. In cardiac fibrosis associated with post-ischemic injury of the heart, EndMT, which involves both the endocardium (the inner endothelial layer of the heart) and the microvascular endothelium of the heart, has been demonstrated to play a key role in contributing to the emergence of newly formed fibroblasts (45) (Figure 4). In tissue culture models of EndMT, TGF- $\beta 1$ induces EndMT of capillary endothelial cells and the loss of endothelial markers, such CD31 and integrin $\alpha \mathrm{V} \beta 3$, as well as the acquisition of fibroblast- and myofibroblast-specific markers such as FSP1, $\alpha$-SMA, DDR2, collagen I, and vimentin. While not yet documented, it is plausible that many of the molecular regulators of EMTs also play critical roles in orchestrating EndMTs.

In one analysis (1), lineage-tagging experiments and bone marrow transplant studies demonstrated that during the course of kidney fibrosis in mice, about $12 \%$ of fibroblasts are derived from the bone marrow and about $30 \%$ are derived via EMT from the tubular epithelial cells of the kidney. In addition, it has been shown more recently that in kidney fibrosis about $35 \%$ of fibroblasts are derived via EndMT from the endothelial cells normally residing within the kidney. The remaining portions are speculated to arise via activation of resident fibroblasts or other mesenchymal cells, such as perivascular smooth muscle cells/ pericytes and fibrocytes (53) in the circulation (Figure 4). It is likely that these proportions may vary dramatically, depending on the stage of fibrosis, the organ, and the particular experimental model being studied.

This complex pathologic process may reflect the developmental origins of the kidney. Thus, the entire epithelium of the kidney, including the tubular epithelial cells, is derived from the intermediate mesoderm during the development of the urogenital system. More specifically, a mesenchyme condenses over the ureteric bud (derived from the endoderm) to give rise to the glomerular and tubular epithelial structures of the kidney (54), doing so via a MET. This hints at a peculiar aspect of renal biology: by retaining some imprint of their mesenchymal origins, kidney epithelial cells may be particularly prone to return to this state, via the EMTs that occur in response to inflammatory stress and lead to pathologic fibrosis $(55,56)$.

Inflammatory injury to the mouse kidney can result in the recruitment of a diverse array of cells that can trigger an EMT through their release of growth factors, such as TGF- $\beta$, PDGF, EGF, and FGF-2 (57). Most prominent among these cells are macrophages and activated resident fibroblasts that accumulate at the site of injury and release these growth factors. In addition, these cells release chemokines and MMPs, notably MMP-2, MMP-3, and MMP-9. Epithelial cells come under the influence of these signaling molecules and, acting together with the inflammatory cells, induce basement membrane damage and focal degradation of type IV collagen and laminin (57). Delaminated epithelial cells may then migrate toward the interstitial area (the space between epithelial layers) under the influence of gradients of growth factors and other chemoattractants (57). This initial recruitment of epithelial cells into an EMT can be inhibited by blocking the expression of MMP-9 through the disruption of tissue plasminogen activator (tPA) (58). Other studies have also demonstrated that HGF can decrease levels of TGF- $\beta$, restore TGF- $\beta$-mediated loss of E-cadherin, and potentially decrease amounts of active MMP-9 (59). $\beta 1$ integrin and integrin-linked kinase (ILK) are also identified as important mediators of the TGF- $\beta$-induced EMT associated with tubular epithelial cells 
A

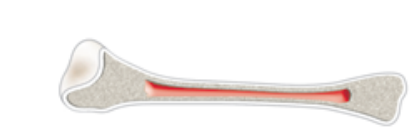

Bone marrow-derived

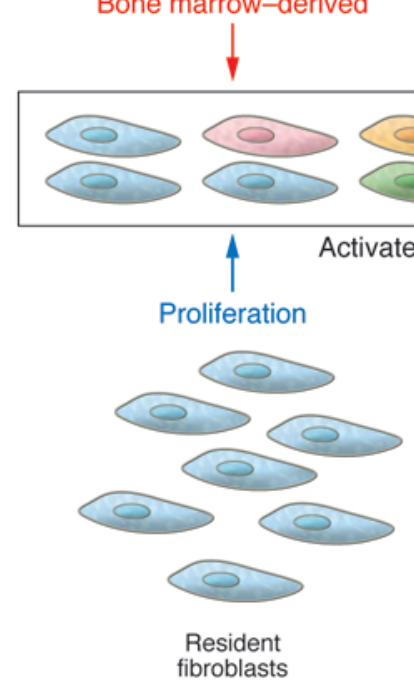

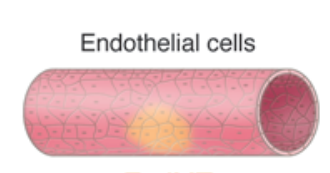

EndMT

$\downarrow$

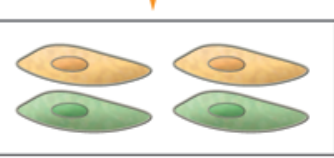

oblasts

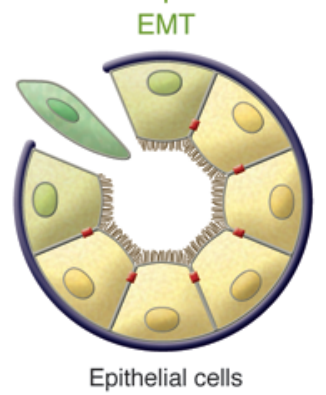

B

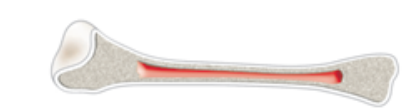

Bone marrow-derived
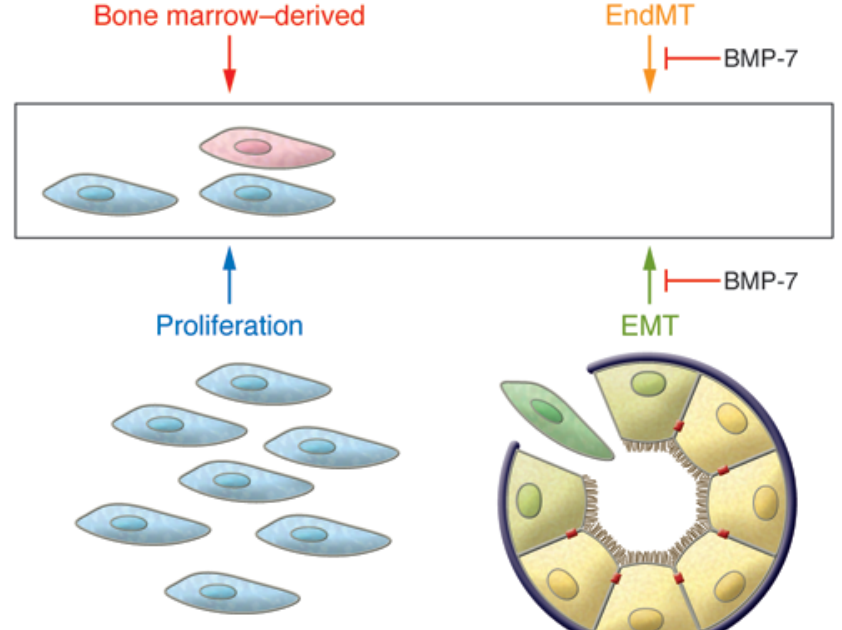

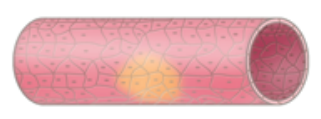

EndMT

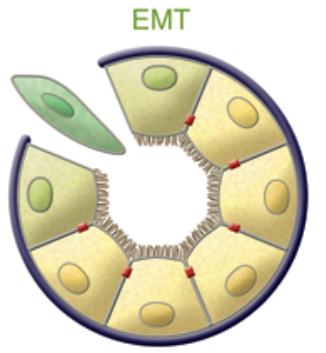

Figure 4

Origin of fibroblasts during fibrosis and its reversal by BMP-7. (A) Different sources of fibroblasts in organ fibrosis. Four possible mechanisms are depicted. One study suggests that about $12 \%$ of fibroblasts are from bone marrow, about $30 \%$ can arise via local EMT involving tubular epithelial cells under inflammatory stress, and about $35 \%$ are from EndMT (1). The remaining percentage likely emerge via proliferation of the resident fibroblasts and other still unidentified sources. (B) Systemic treatment of mice with renal fibrosis with recombinant human BMP-7 reverses renal disease due to severe attenuation of the formation of EMT- and EndMT-derived fibroblasts.

(60). TGF- $\beta$ induces EMT via both a Smad2/3-dependent pathway and a MAPK-dependent pathway. Recent experiments have also demonstrated a key role for the E-cadherin/ $\beta$-catenin signaling axis for EMT involving epithelial cells $(61,62)$.

The significance of TGF- $\beta$-induced EMT for progression of organ fibrosis has been demonstrated in studies using BMP-7, an antagonist of TGF- $\beta$ signaling, in mouse models of kidney, liver, billiard tract, lung, and intestinal fibrosis $(51,63)$. BMP-7 functions as an endogenous inhibitor of TGF- $\beta$-induced EMT $(51,63)$ (Figure 4). Among other effects, it reverses the TGF- $\beta$-induced loss of the key epithelial protein, E-cadherin (63). Restoration of E-cadherin levels by BMP-7 is mediated via its cognate receptors, activinlike kinase-2/3/6 (ALK-2/3/6), and the Smad4/5 downstream transcription factors (63). Systemic administration of recombinant BMP-7 to mice with severe fibrosis resulted in reversal of EMT and repair of damaged epithelial structures, with repopulation of healthy epithelial cells, all presumably mediated via a MET (63). This reversal was also associated with restoration of organ function, a substantial decrease in FSP $1^{+}$and $\alpha$-SMA ${ }^{+}$interstitial fibroblasts, and the de novo activation of BMP-7 signaling (63).

Studies using fibrosis tissue from humans have also demonstrated EMT (50). In a study of 133 patients with kidney fibrosis, an EMT was demonstrated in a substantial number of the samples, as evaluated using double labeling of the tubular epithelial cells with cytokeratin, vimentin, $\alpha$-SMA, or zona occludens 1 (ZO-1). Similarly, in patients with Crohn disease, an EMT was demonstrated in areas of fibrosis in the colon (64). Such studies should provide the necessary confidence to develop novel therapeutic interventions in order to suppress EMTs and potentially reverse organ fibrosis.

\section{Type 3 EMT: EMT associated with cancer progression and metastasis}

Excessive epithelial cell proliferation and angiogenesis are hallmarks of the initiation and early growth of primary epithelial cancers (65). The subsequent acquisition of invasiveness, initially manifest by invasion through the basement membrane, is thought to herald the onset of the last stages of the multi-step process that leads eventually to metastatic dissemination, with life-threatening consequences. The genetic controls and biochemical mechanisms underlying the acquisition of the invasive phenotype and the subsequent systemic spread of the cancer cell have been areas of intensive research. In many of these studies, activation of an EMT program has been proposed as the critical mechanism for the acquisition of malignant phenotypes by epithelial cancer cells (66).

Many mouse studies and cell culture experiments have demonstrated that carcinoma cells can acquire a mesenchymal phenotype and express mesenchymal markers such as $\alpha$-SMA, FSP1, vimentin, and desmin (67). These cells typically are seen at the invasive front of primary tumors and are considered to be the cells that eventually enter into subsequent steps of the invasion-metastasis cascade, i.e., intravasation, transport through the circulation, extravasation, formation of micrometastases, and ultimately colonization (the growth of small colonies into macroscopic metastases) $(66,68,69)$.

An apparent paradox comes from the observation that the EMT-derived migratory cancer cells typically establish secondary colonies at distant sites that resemble, at the histopathological level, the primary tumor from which they arose; accordingly, they no longer exhibit the mesenchymal phenotypes ascribed to metastasizing carcinoma cells. Reconciling this behavior with 


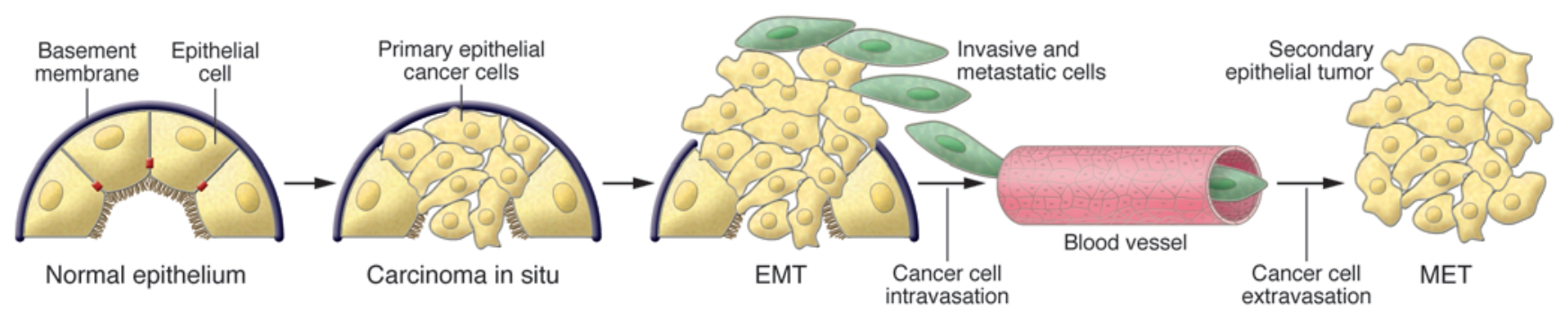

Figure 5

Contribution of EMT to cancer progression. Progression from normal epithelium to invasive carcinoma goes through several stages. The invasive carcinoma stage involves epithelial cells losing their polarity and detaching from the basement membrane. The composition of the basement membrane also changes, altering cell-ECM interactions and signaling networks. The next step involves EMT and an angiogenic switch, facilitating the malignant phase of tumor growth. Progression from this stage to metastatic cancer also involves EMTs, enabling cancer cells to enter the circulation and exit the blood stream at a remote site, where they may form micro- and macro-metastases, which may involve METs and thus a reversion to an epithelial phenotype.

the proposed role of EMT as a facilitator of metastatic dissemination requires the additional notion that metastasizing cancer cells must shed their mesenchymal phenotype via a MET during the course of secondary tumor formation (70). The tendency of disseminated cancer cells to undergo MET likely reflects the local microenvironments that they encounter after extravasation into the parenchyma of a distant organ, quite possibly the absence of the heterotypic signals they experienced in the primary tumor that were responsible for inducing the EMT in the first place $(66,71$, 72). These considerations indicate that induction of an EMT is likely to be a centrally important mechanism for the progression of carcinomas to a metastatic stage and implicates MET during the subsequent colonization process (Figure 5). However, many steps of this mechanistic model still require direct experimental validation. Moreover, it remains unclear at present whether these phenomena and molecular mechanisms relate to and explain the metastatic dissemination of non-epithelial cancer cells.

The full spectrum of signaling agents that contribute to EMTs of carcinoma cells remains unclear. One suggestion is that the genetic and epigenetic alterations undergone by cancer cells during the course of primary tumor formation render them especially responsive to EMT-inducing heterotypic signals originating in the tumor-associated stroma. Oncogenes induce senescence, and recent studies suggest that cancer cell EMTs may also play a role in preventing senescence induced by oncogenes, thereby facilitating subsequent aggressive dissemination (73-75). In the case of many carcinomas, EMT-inducing signals emanating from the tumor-associated stroma, notably HGF, EGF, PDGF, and TGF- $\beta$, appear to be responsible for the induction or functional activation in cancer cells of a series of EMT-inducing transcription factors, notably Snail, Slug, zinc finger E-box binding homeobox 1 (ZEB1), Twist, Goosecoid, and FOXC2 (66, 71, 76-79). Once expressed and activated, each of these transcription factors can act pleiotropically to choreograph the complex EMT program, more often than not with the help of other members of this cohort of transcription factors. The actual implementation by these cells of their EMT program depends on a series of intracellular signaling networks involving, among other signaltransducing proteins, ERK, MAPK, PI3K, Akt, Smads, RhoB, $\beta$-catenin, lymphoid enhancer binding factor (LEF), Ras, and c-Fos as well as cell surface proteins such as $\beta 4$ integrins, $\alpha 5 \beta 1$ integrin, and $\alpha \mathrm{V} \beta 6$ integrin (80). Activation of EMT programs is also facilitated by the disruption of cell-cell adherens junctions and the cell-ECM adhesions mediated by integrins (67, 75, 81-86).

TGF- $\beta$ is an important suppressor of epithelial cell proliferation and thus primary tumorigenesis. However, it is now clear that in certain contexts it can also serve as a positive regulator of tumor progression and metastasis (87-89). Thus, in vitro studies have demonstrated that TGF- $\beta$ can induce an EMT in certain types of cancer cells (90). Two possible signaling pathways have been identified as mediators of TGF- $\beta$-induced EMT. The first of these involves Smad proteins, which mediate TGF- $\beta$ action to induce EMTs via the ALK-5 receptor $(51,91-95)$. Smad-mediated signaling induced by TGF- $\beta$ facilitates motility. Inhibitory Smads modulate differential effects of relevant transcription factors and cytoplasmic kinases and induce the autocrine production of TGF- $\beta$, which can further reinforce and amplify the EMT program $(91,92$, $96,97)$. Signaling pathways that mediate the action of $\beta$-catenin and LEF also cooperate with Smads $(61,98)$ in inducing an EMT $(61,99,100)$. In this regard, the involvement of LEF and $\beta$-catenin in PDGF-induced EMT was recently described (98). These studies collectively demonstrate that the TGF- $\beta / \mathrm{Smad} / \mathrm{LEF} / \mathrm{PDGF}$ axis is an important inducer of an EMT phenotype in cancer.

Evidence for the involvement of a second TGF- $\beta$-induced pathway in EMT is also compelling. More specifically, some data indicate that $\mathrm{p} 38$ MAPK and RhoA mediate an autocrine TGF- $\beta$ induced EMT in NMuMG mouse mammary epithelial cells (96, 101). This process also requires integrin $\beta 1$-mediated signaling and the activation of latent TGF- $\beta$ by $\alpha V \beta 6$ integrin $(96,101)$. Fibulin- 5 , an ECM molecule, augments TGF- $\beta$-induced EMT in a MAPKdependent mechanism (102). TGF- $\beta$ can induce an EMT in Rastransformed hepatocytes, mammary epithelial cells (via MAPK), and MDCK cells; at the same time, Ras-activated PI3K inhibits TGF- $\beta$-induced apoptosis to facilitate this transition (103-106). Evidence for these connections comes from observations that ERK/MAPK and PI3K/Akt pathways mediate Ras mutant-induced EMT, and that such an EMT is reversed by either wild-type Ras or MAPK kinase 1 (MEK1) inhibitors (106). In this regard, Raf also mediates TGF- $\beta$-induced EMT and promotes invasiveness of cancer cells. In mouse models of skin carcinoma and human colon cancer, the absence of TGF- $\beta$ receptor expression actually confers better prognosis $(107,108)$. The connection between inflammation and EMT was demonstrated when COX-2 was shown to inactivate Smad signaling and enhance EMT stimulated by TGF- $\beta$ through 
a $\mathrm{PGE}_{2}$-dependent mechanism (109). Changes in the expression of certain cell polarity proteins may also play an important role in TGF- $\beta$-induced EMT, since evidence of a role for partitioningdefective protein 6 (Par6) in this process is emerging $(66,110)$.

The connection between loss of E-cadherin expression by cancer cells and passage through an EMT has been established by many studies $(111,112)$. For example, induction of the $c$-Fos oncogene in normal mouse mammary epithelial cell lines induces an EMT and is associated with a decrease in E-cadherin expression (99). Moreover, epithelial cell adhesion complexes reorganize and cell proliferation is suppressed when the full-length or the cytoplasmic portion of E-cadherin (containing the $\beta$-catenin binding site) is ectopically expressed in cells that have passed through an EMT, causing such cells to lose their mesenchymal phenotype $(99,113)$. Sequestration of $\beta$-catenin in the cytoplasm is important for the preservation of epithelial features of cancer cells, and acquisition of the mesenchymal phenotype correlates with the movement of $\beta$-catenin to the nucleus, where it becomes part of Tcf/LEF complexes $(100,114)$. Such $\beta$-catenin accumulation in the nucleus, which is often associated with loss of E-cadherin expression, correlates with susceptibility to enter into an EMT and acquisition of an invasive phenotype $(61,66)$. Thus, cells that lose cell surface E-cadherin become more responsive to induction of an EMT by various growth factors (61).

Some studies have demonstrated that the epigenetic control of E-cadherin and $\beta$-catenin/LEF activity is important in establishing the metastatic potential of cancer cells (115-118). Cell lines that lack E-cadherin show increased tumorigenicity and metastasis when transferred into immunodeficient mice (118). E-cadherin expression levels vary dramatically in different human tumors, and an inverse relationship between levels of E-cadherin and patient survival has been documented (117). In this regard, mutations in the E-cadherin gene have been identified in cancer cells, making them more susceptible to EMT and metastasis $(115,116)$. A more thorough analysis of such mutations and their correlation to metastatic progression is still required.

The central role played by E-cadherin loss in the EMT program is further illustrated by the actions of several EMT-inducing transcription factors that facilitate acquisition of a mesenchymal phenotype, such as Snail and Slug, as well as those encoding two key zinc finger-containing basic helix-loop-helix transcription factors, survival of motor neuron protein interacting protein 1 (SIP1) and E12 (also known as E47-E2A). These transcription factors are induced by TGF- $\beta$ exposure and, once expressed, repress E-cadherin expression (78). Snail also facilitates an invasive phenotype in mice (31). Loss of E-cadherin promotes Wnt signaling and is associated with high levels of Snail in the nucleus (119). SIP1 represses E-cadherin expression and binds, along with Snail, to the E-cadherin promoter in an overlapping fashion $(120,121)$. The expression of Snail and E-cadherin correlates inversely with the prognosis of patients suffering from breast cancer or oral squamous cell carcinoma $(119,122)$. The use of gene expression analyses to compare expression of genes in metastatic and non-metastatic mouse breast cancer cell lines has led to the identification of Twist and Goosecoid as important genes that facilitate EMT and induce metastasis $(85,123)$. Some have reported that matrix-degrading enzymes such as MMP-3 facilitate EMT by inducing genomic instability via Rac1b and ROS (124).

Noncoding microRNAs are also components of the cellular signaling circuitry that regulates the EMT program. For example, microRNA 200 (miR200) and miR205 inhibit the repressors of E-cadherin expression, ZEB1 and ZEB2, and thereby help in maintaining the epithelial cell phenotype (125-129). In breast carcinoma, a loss of miR200 correlates with increased expression of vimentin and a decrease in the levels of E-cadherin in cancer cells (125-127, 129). Acting in the opposite direction, miR2 1 is upregulated in many cancers and facilitates TGF- $\beta$-induced EMT (130). Interestingly, CD $44^{\text {hi }} \mathrm{CD} 20^{\text {lo }}$ cells purified from normal and malignant breast cancer tissue exhibit features of an EMT, and human cancer cells induced to undergo EMT exhibit stem cell-like properties and increased metastatic potential (84). Therefore, EMT may play a role in the generation of high-grade invasive cells with stem cell-like features, and the latter phenotype, which includes self-renewal potential, may facilitate the formation of secondary tumors by disseminating cancer cells, a notion that still requires direct demonstration.

\section{Perspective}

It is now clear that EMTs occur in three distinct biological settings. While the outcome is the generation of motile cells of mesenchymal phenotype, the mechanisms of EMT induction and progression vary dramatically from one setting to another. The EMTs associated with implantation, embryogenesis, and organ development (type 1 EMTs) are driven by the evolutionary need to remodel and diversify tissue in order to enable proper morphogenesis and generate a functional organism. Such EMTs occur transiently and are not associated with inflammation, fibrosis, and systemic dissemination. Type 2 EMTs are associated with tissue regeneration and fibrosis, and they depend on inflammation-inducing injuries for their initiation and continued occurrence. Accordingly, these EMTs continue to occur until the provoking injuries or infections are removed and, in the case of injuries, the tissue is repaired. Type 2 EMTs generate activated mesenchymal cells, notably myofibroblasts that produce excessive amounts of collagen-rich ECM. Type 3 EMTs occur in the context of tumor growth and cancer progression, when cancer cells at the invasive front of the tumors convert to a mesenchymal phenotype. The induction of type 3 EMTs is facilitated by the genomic alterations acquired by cancer cells and these EMTs generate cells with invasive properties that enable them to move into the blood stream and spread systemically to other organs. Future research will surely focus on uncovering the molecular similarities and differences among the EMT programs that occur in the three distinct settings.

EMT research in the next few years promises to be exciting, as new mouse models and molecular probes are identified to address important, still-unanswered questions. For example, what are the identities of the EMT-inducing microenvironmental signals? What is the nature of the changes in cells that render them responsive to such signals? And what signaling machinery within epithelial cells orchestrates the various EMT programs?

\section{Acknowledgments}

Research work in the Kalluri laboratory is supported by NIH grants DK55001, DK62978, DK61688, AA13913, and CA125550 and by research funds from the Division of Matrix Biology at Beth Israel Deaconess Medical Center. Research work in the Weinberg laboratory is supported by NIH grants U54 CA12515 and R01 CA078461 and the Ludwig Center for Molecular Oncology at Massachusetts Institute of Technology.

Address correspondence to: Raghu Kalluri, Harvard Medical School, Division of Matrix Biology, Beth Israel Deaconess Medical Center, 3 Blackfan Circle, CLS 11801, Boston, Massachusetts 02215, USA. Phone: (617) 667-0445; Fax: (617) 975-5663; E-mail: rkalluri@bidmc.harvard.edu. 
1. Kalluri, R., and Neilson, E.G. 2003. Epithelialmesenchymal transition and its implications for fibrosis. J. Clin. Invest. 112:1776-1784.

2. Hay, E.D. 1995. An overview of epithelio-mesenchymal transformation. Acta Anat. (Basel). 154:8-20.

3. Lipschutz, J.H. 1998. Molecular development of the kidney: a review of the results of gene disruption studies. Am. J. Kidney Dis. 31:383-397.

4. Rothenpieler, U.W., and Dressler, G.R. 1993. Pax-2 is required for mesenchyme-to-epithelium conversion during kidney development. Development. 119:711-720.

5. Hogan, B.L., and Kolodziej, P.A. 2002. Organogenesis: molecular mechanisms of tubulogenesis. Nat. Rev. Genet. 3:513-523.

6. Virchow, R. 1858. Die Cellularpathologie in ibrer Begruendung auf physiologische und pathologische Gewebelehre. August Hirschwald. Berlin, Germany.

7. Lee, J.M., Dedhar, S., Kalluri, R., and Thompson, E.W. 2006. The epithelial-mesenchymal transition: new insights in signaling, development, and disease. J. Cell Biol. 172:973-981.

8. Zeisberg, M., and Neilson, E.G. 2009. Biomarkers for epithelial-mesenchymal transitions. J. Clin. Invest. 119:1429-1437.

9. Vicovac, L., and Aplin, J.D. 1996. Epithelialmesenchymal transition during trophoblast differentiation. Acta Anat. (Basel). 156:202-216.

10. Aplin, J.D., Haigh, T., Vicovac, L., Church, H.J., and Jones, C.J. 1998. Anchorage in the developing placenta: an overlooked determinant of pregnancy outcome? Hum. Fertil. (Camb.). 1:75-79.

11. Bischof, P., et al. 2006. Implantation of the human embryo: research lines and models. From the implantation research network 'Fruitful'. Gynecol. Obstet. Invest. 62:206-216.

12. Acloque, H., Adams, M.S., Fishwick, K., Bronner-Fraser, M., and Nieto, M.A. 2009. Epithelial-mesenchymal transitions: the importance of changing cell state in development and disease. J. Clin. Invest. 119:1438-1449.

13. Hay, E.D. 1990. Role of cell-matrix contacts in cell migration and epithelial-mesenchymal transformation. Cell Differ. Dev. 32:367-375.

14. Thiery, J.P., and Sleeman, J.P. 2006. Complex networks orchestrate epithelial-mesenchymal transitions. Nat. Rev. Mol. Cell Biol. 7:131-142.

15. Hay, E.D. 2005. The mesenchymal cell, its role in the embryo, and the remarkable signaling mechanisms that create it. Dev. Dyn. 233:706-720.

16. Skromne, I., and Stern, C.D. 2001. Interactions between Wnt and $\mathrm{Vg} 1$ signalling pathways initiate primitive streak formation in the chick embryo. Development. 128:2915-2927.

17. Liu, P., et al. 1999. Requirement for Wnt3 in vertebrate axis formation. Nat. Genet. 22:361-365.

18. Popperl, H., et al. 1997. Misexpression of Cwnt8C in the mouse induces an ectopic embryonic axis and causes a truncation of the anterior neuroectoderm. Development. 124:2997-3005.

19. Thomas, P., Brickman, J.M., Popperl, H., Krumlauf, R., and Beddington, R.S. 1997. Axis duplication and anterior identity in the mouse embryo. Cold Spring Harb. Symp. Quant. Biol. 62:115-125.

20. Chea, H.K., Wright, C.V., and Swalla, B.J. 2005. Nodal signaling and the evolution of deuterostome gastrulation. Dev. Dyn. 234:269-278.

21. Skromne, I., and Stern, C.D. 2002. A hierarchy of gene expression accompanying induction of the primitive streak by Vg1 in the chick embryo. Mech. Dev. 114:115-118.

22. Collignon, J., Varlet, I., and Robertson, E.J. 1996. Relationship between asymmetric nodal expression and the direction of embryonic turning. Nature. 381:155-158.

23. Varlet, I., Collignon, J., and Robertson, E.J. 1997. nodal expression in the primitive endoderm is required for specification of the anterior axis during mouse gastrulation. Development. 124:1033-1044.

24. Shah, S.B., et al. 1997. Misexpression of chick Vg1 in the marginal zone induces primitive streak formation. Development. 124:5127-5138.

25. Ciruna, B., and Rossant, J. 2001. FGF signaling regulates mesoderm cell fate specification and morphogenetic movement at the primitive streak. Dev. Cell. 1:37-49.

26. Rossant, J., Ciruna, B., and Partanen, J. 1997. FGF signaling in mouse gastrulation and anteroposterior patterning. Cold Spring Harb. Symp. Quant. Biol. 62:127-133.

27. Zhao, H., et al. 2006. Fibroblast growth factor receptor 1 (Fgfr 1 ) is not essential for lens fiber differentiation in mice. Mol. Vis. 12:15-25.

28. Perea-Gomez, A., et al. 2002. Nodal antagonists in the anterior visceral endoderm prevent the formation of multiple primitive streaks. Dev. Cell. 3:745-756.

29. Lindsley, R.C., et al. 2008. Mesp1 coordinately regulates cardiovascular fate restriction and epithelial-mesenchymal transition in differentiating ESCs. Cell Stem Cell. 3:55-68.

30. Arnold, S.J., Hofmann, U.K., Bikoff, E.K., and Robertson, E.J. 2008. Pivotal roles for eomesodermin during axis formation, epithelium-to-mesenchyme transition and endoderm specification in the mouse. Development. 135:501-511.

31. Nieto, M.A. 2002. The snail superfamily of zincfinger transcription factors. Nat. Rev. Mol. Cell Biol. 3:155-166.

32. Ikenouchi, J., Sasaki, H., Tsukita, S., and Furuse, M. 2008. Loss of occludin affects tricellular localization of tricellulin. Mol. Biol. Cell. 19:4687-4693.

33. Cavatorta, A.L., Giri, A.A., Banks, L., and Gardiol, D. 2008. Cloning and functional analysis of the promoter region of the human Disc large gene. Gene. 424:87-95

34. Whiteman, E.L., Liu, C.J., Fearon, E.R., and Margolis, B. 2008. The transcription factor snail represses Crumbs3 expression and disrupts apico-basal polarity complexes. Oncogene. 27:3875-3879.

35. Duband, J.L., and Thiery, J.P. 1982. Appearance and distribution of fibronectin during chick embryo gastrulation and neurulation. Dev. Biol. 94:337-350.

36. Sauka-Spengler, T., and Bronner-Fraser, M. 2008. A gene regulatory network orchestrates neural crest formation. Nat. Rev. Mol. Cell Biol. 9:557-568.

37. Knecht, A.K., and Bronner-Fraser, M. 2002. Induction of the neural crest: a multigene process. Nat. Rev. Genet. 3:453-461.

38. Liem, K.F., Jr., Jessell, T.M., and Briscoe, J. 2000. Regulation of the neural patterning activity of sonic hedgehog by secreted BMP inhibitors expressed by notochord and somites. Development. 127:4855-4866.

39. Villanueva, S., Glavic, A., Ruiz, P., and Mayor, R. 2002. Posteriorization by FGF, Wnt, and retinoic acid is required for neural crest induction. Dev. Biol. 241:289-301.

40. Karafiat, V., Dvorakova, M., Pajer, P., Cermak, V., and Dvorak, M. 2007. Melanocyte fate in neural crest is triggered by Myb proteins through activation of c-kit. Cell Mol. Life Sci. 64:2975-2984.

41. Burstyn-Cohen, T., Stanleigh, J., Sela-Donenfeld, D., and Kalcheim, C. 2004. Canonical Wnt activity regulates trunk neural crest delamination linking $\mathrm{BMP} /$ noggin signaling with G1/S transition. Development. 131:5327-5339.

42. Sela-Donenfeld, D., and Kalcheim, C. 2002. Localized BMP4-noggin interactions generate the dynamic patterning of noggin expression in somites. Dev. Biol. 246:311-328.

43. Thiery, J.P. 2003. Epithelial-mesenchymal transitions in development and pathologies. Curr. Opin. Cell Biol. 15:740-746.

44. Potenta, S., Zeisberg, E., and Kalluri, R. 2008. The role of endothelial-to-mesenchymal transition in cancer progression. Br. J. Cancer. 99:1375-1379.
45. Zeisberg, E.M., et al. 2007. Endothelial-tomesenchymal transition contributes to cardiac fibrosis. Nat. Med. 13:952-961.

46. Zeisberg, M., et al. 2007. Fibroblasts derive from hepatocytes in liver fibrosis via epithelial to mesenchymal transition. J. Biol. Chem. 282:23337-23347.

47. Kim, K.K., et al. 2006. Alveolar epithelial cell mesenchymal transition develops in vivo during pulmonary fibrosis and is regulated by the extracellular matrix. Proc. Natl. Acad. Sci. U. S. A. 103:13180-13185.

48. Strutz, F., et al. 1995. Identification and characterization of a fibroblast marker: FSP1. J. Cell Biol. 130:393-405.

49. Okada, H., Danoff, T.M., Kalluri, R., and Neilson, E.G. 1997. Early role of Fsp1 in epithelial-mesenchymal transformation. Am. J. Physiol. 273:F563-F574.

50. Rastaldi, M.P., et al. 2002. Epithelial-mesenchymal transition of tubular epithelial cells in human renal biopsies. Kidney Int. 62:137-146.

51. Zeisberg, M., et al. 2003. BMP-7 counteracts TGFbeta1-induced epithelial-to-mesenchymal transition and reverses chronic renal injury. Nat. Med. 9:964-968.

52. Okada, H., Strutz, F., Danoff, T.M., Kalluri, R., and Neilson, E.G. 1996. Possible mechanisms of renal fibrosis. Contrib. Nephrol. 118:147-154.

53. Zavadil, J., and Bottinger, E.P. 2005. TGF-beta and epithelial-to-mesenchymal transitions. Oncogene. 24:5764-5774

54. Ekblom, P. 1996. Genetics of kidney development. Curr. Opin. Nephrol. Hypertens. 5:282-287.

55. Aufderheide, E., Chiquet-Ehrismann, R., and Ekblom, P. 1987. Epithelial-mesenchymal interactions in the developing kidney lead to expression of tenascin in the mesenchyme. J. Cell Biol. 105:599-608.

56. Ivanova, L., Butt, M.J., and Matsell, D.G. 2008. Mesenchymal transition in kidney collecting duct epithelial cells. Am. J. Physiol. Renal Physiol. 294:F1238-F1248.

57. Strutz, F., et al. 2002. Role of basic fibroblast growth factor-2 in epithelial-mesenchymal transformation. Kidney Int. 61:1714-1728.

58. Yang, J., et al. 2002. Disruption of tissue-type plasminogen activator gene in mice reduces renal interstitial fibrosis in obstructive nephropathy. J. Clin. Invest. 110:1525-1538.

59. Yang, J., and Liu, Y. 2002. Blockage of tubular epithelial to myofibroblast transition by hepatocyte growth factor prevents renal interstitial fibrosis. J. Am. Soc. Nephrol. 13:96-107.

60. Li, Y., Yang, J., Dai, C., Wu, C., and Liu, Y. 2003. Role for integrin-linked kinase in mediating tubular epithelial to mesenchymal transition and renal interstitial fibrogenesis. J. Clin. Invest. 112:503-516.

61. Kim, K., Lu, Z., and Hay, E.D. 2002. Direct evidence for a role of beta-catenin/LEF-1 signaling pathway in induction of EMT. Cell Biol. Int. 26:463-476.

62. Nawshad, A., Lagamba, D., Polad, A., and Hay, E.D. 2005. Transforming growth factor-beta signaling during epithelial-mesenchymal transformation: implications for embryogenesis and tumor metastasis. Cells Tissues Organs. 179:11-23.

63. Zeisberg, M., et al. 2003. Bone morphogenic protein-7 inhibits progression of chronic renal fibrosis associated with two genetic mouse models. Am. J. Physiol. Renal Physiol. 285:F1060-F1067.

64. Bataille, F., et al. 2008. Evidence for a role of epithelial mesenchymal transition during pathogenesis of fistulae in Crohn's disease. Inflamm. Bowel Dis. 14:1514-1527.

65. Hanahan, D., and Weinberg, R.A. 2000. The hallmarks of cancer. Cell. 100:57-70.

66. Thiery,J.P. 2002. Epithelial-mesenchymal transitions in tumour progression. Nat. Rev. Cancer. 2:442-454.

67. Yang, J., and Weinberg, R.A. 2008. Epithelialmesenchymal transition: at the crossroads of development and tumor metastasis. Dev. Cell. 14:818-829. 68. Fidler, I.J., and Poste, G. 2008. The "seed and soil" 
hypothesis revisited. Lancet Oncol. 9:808.

69. Brabletz, T., et al. 2001. Variable beta-catenin expression in colorectal cancers indicates tumor progression driven by the tumor environment. Proc. Natl. Acad. Sci. U. S. A. 98:10356-10361.

70. Zeisberg, M., Shah, A.A., and Kalluri, R. 2005. Bone morphogenic protein-7 induces mesenchymal to epithelial transition in adult renal fibroblasts and facilitates regeneration of injured kidney. J. Biol. Chem. 280:8094-8100.

71. Jechlinger, M., Grunert, S., and Beug, H. 2002. Mechanisms in epithelial plasticity and metastasis: insights from 3D cultures and expression profiling. J. Mammary Gland Biol. Neoplasia. 7:415-432.

72. Bissell, M.J., Radisky, D.C., Rizki, A., Weaver, V.M. and Petersen, O.W. 2002. The organizing principle: microenvironmental influences in the normal and malignant breast. Differentiation. 70:537-546.

73. Smit, M.A., and Peeper, D.S. 2008. Deregulating EMT and senescence: double impact by a single twist. Cancer Cell. 14:5-7.

74. Ansieau, S., et al. 2008. Induction of EMT by twist proteins as a collateral effect of tumor-promoting inactivation of premature senescence. Cancer Cell. 14:79-89.

75. Weinberg, R.A. 2008. Twisted epithelial-mesenchymal transition blocks senescence. Nat. Cell Biol. 10:1021-1023

76. Shi, Y., and Massague, J. 2003. Mechanisms of TGFbeta signaling from cell membrane to the nucleus. Cell. 113:685-700.

77. Niessen, K., et al. 2008. Slug is a direct Notch target required for initiation of cardiac cushion cellular ization. J. Cell Biol. 182:315-325.

78. Medici, D., Hay, E.D., and Olsen, B.R. 2008. Snail and Slug promote epithelial-mesenchymal transition through beta-catenin-T-cell factor-4-dependent expression of transforming growth factorbeta3. Mol. Biol. Cell. 19:4875-4887.

79. Kokudo, T., et al. 2008. Snail is required for TGF $\{$ beta $\}$-induced endothelial-mesenchymal transition of embryonic stem cell-derived endothelial cells. J. Cell. Sci. 121:3317-3324.

80. Tse, J.C., and Kalluri, R. 2007. Mechanisms of metastasis: epithelial-to-mesenchymal transition and contribution of tumor microenvironment. J. Cell. Biochem. 101:816-829.

81. Gupta, P.B., Mani, S., Yang, J., Hartwell, K., and Weinberg, R.A. 2005. The evolving portrait of cancer metastasis. Cold Spring Harb. Symp. Quant. Biol. 70:291-297.

82. Yang, J., Mani, S.A., and Weinberg, R.A. 2006. Exploring a new twist on tumor metastasis. Cancer Res. 66:4549-4552.

83. Mani, S.A., et al. 2007. Mesenchyme Forkhead 1 (FOXC2) plays a key role in metastasis and is associated with aggressive basal-like breast cancers. Proc. Natl. Acad. Sci. U. S. A. 104:10069-10074.

84. Mani, S.A., et al. 2008. The epithelial-mesenchymal transition generates cells with properties of stem cells. Cell. 133:704-715.

85. Hartwell, K.A., et al. 2006. The Spemann organizer gene, Goosecoid, promotes tumor metastasis. Proc. Natl. Acad. Sci. U. S. A. 103:18969-18974.

86. Taki, M., Verschueren, K., Yokoyama, K., Nagayama, M., and Kamata, N. 2006. Involvement of Ets-1 transcription factor in inducing matrix metalloproteinase- 2 expression by epithelial-mesenchymal transition in human squamous carcinoma cells. Int J. Oncol. 28:487-496.

87. Bierie, B., and Moses, H.L. 2006. Tumour microenvironment: TGFbeta: the molecular Jekyll and Hyde of cancer. Nat. Rev. Cancer. 6:506-520.

88. Oft, M., Heider, K.H., and Beug, H. 1998. TGFbeta signaling is necessary for carcinoma cell invasiveness and metastasis. Curr. Biol. 8:1243-1252.

89. Hata, A., Shi, Y., and Massague, J. 1998. TGF-beta signaling and cancer: structural and functional consequences of mutations in Smads. Mol. Med. Today.
4:257-262.

90. Song, J. 2007. EMT or apoptosis: a decision for TGF-beta. Cell Res. 17:289-290.

91. Miyazono, K., ten Dijke, P., and Heldin, C.H. 2000. TGF-beta signaling by Smad proteins. Adv. Immunol. 75:115-157.

92. Derynck, R., Akhurst, R.J., and Balmain, A. 2001. TGF-beta signaling in tumor suppression and cancer progression. Nat. Genet. 29:117-129.

93. Heldin, C.H., Miyazono, K., and ten Dijke, P. 1997. TGF-beta signalling from cell membrane to nucleus through SMAD proteins. Nature. 390:465-471.

94. Roberts, A.B., et al. 2006. Smad3 is key to TGFbeta-mediated epithelial-to-mesenchymal transition, fibrosis, tumor suppression and metastasis. Cytokine Growth Factor Rev. 17:19-27.

95. Piek, E., Moustakas, A., Kurisaki, A., Heldin, C.H., and ten Dijke, P. 1999. TGF-(beta) type I receptor/ALK-5 and Smad proteins mediate epithelial to mesenchymal transdifferentiation in NMuMG breast epithelial cells. J. Cell. Sci. 112:4557-4568.

96. Bhowmick, N.A., et al. 2001. Transforming growth factor-beta 1 mediates epithelial to mesenchymal transdifferentiation through a RhoA-dependent mechanism. Mol. Biol. Cell. 12:27-36.

97. Saika, S., et al. 2004. Transient adenoviral gene transfer of Smad7 prevents injury-induced epithelial-mesenchymal transition of lens epithelium in mice. Lab. Invest. 84:1259-1270.

98. Yang, L., Lin, C., and Liu, Z.R. 2006. P68 RNA helicase mediates PDGF-induced epithelial mesenchymal transition by displacing Axin from beta-catenin. Cell. 127:139-155.

99. Eger, A., Stockinger, A., Schaffhauser, B., Beug, H., and Foisner, R. 2000. Epithelial mesenchymal transition by c-Fos estrogen receptor activation involves nuclear translocation of beta-catenin and upregulation of beta-catenin/lymphoid enhancer binding factor-1 transcriptional activity. J. Cell Biol. 148:173-188.

100.Stockinger, A., Eger, A., Wolf, J., Beug, H., and Foisner, R. 2001. E-cadherin regulates cell growth by modulating proliferation-dependent beta-catenin transcriptional activity. J. Cell Biol. 154:1185-1196.

101.Bhowmick, N.A., Zent, R., Ghiassi, M., McDonnell, M., and Moses, H.L. 2001. Integrin beta 1 signaling is necessary for transforming growth factor-beta activation of p38MAPK and epithelial plasticity. J. Biol. Chem. 276:46707-46713.

102.Lee, Y.H., Albig, A.R., Maryann, R., Schiemann, B.J., and Schiemann, W.P. 2008. Fibulin-5 initiates epithelial-mesenchymal transition (EMT) and enhances EMT induced by TGF-beta in mammary epithelial cells via a MMP-dependent mechanism. Carcinogenesis. 29:2243-2251.

103. Lehmann, K., et al. 2000. Raf induces TGFbeta production while blocking its apoptotic but not invasive responses: a mechanism leading to increased malignancy in epithelial cells. Genes Dev. 14:2610-2622.

104.Gotzmann, J., et al. 2002. Hepatocytes convert to a fibroblastoid phenotype through the cooperation of TGF-beta1 and Ha-Ras: steps towards invasiveness. J. Cell. Sci. 115:1189-1202.

105. Oft, M., et al. 1996. TGF-beta1 and Ha-Ras collaborate in modulating the phenotypic plasticity and invasiveness of epithelial tumor cells. Genes Dev. 10:2462-2477.

106.Janda, E., et al. 2002. Ras and TGF[beta] cooperatively regulate epithelial cell plasticity and metastasis: dissection of Ras signaling pathways. J. Cell Biol. 156:299-313.

107. Cui, W., et al. 1996. TGFbeta1 inhibits the formation of benign skin tumors, but enhances progression to invasive spindle carcinomas in transgenic mice. Cell. 86:531-542.

108. Watanabe, T., et al. 2001. Molecular predictors of survival after adjuvant chemotherapy for colon cancer. N. Engl. J. Med. 344:1196-1206.

109.Neil, J.R., Johnson, K.M., Nemenoff, R.A., and
Schiemann, W.P. 2008. Cox-2 inactivates Smad signaling and enhances EMT stimulated by TGF-beta through a PGE2-dependent mechanisms. Carcinogenesis. 29:2227-2235.

110.Thiery, J.P., and Huang, R. 2005. Linking epithelialmesenchymal transition to the well-known polarity protein Par6. Dev. Cell. 8:456-458.

111.Tepass, U., Truong, K., Godt, D., Ikura, M., and Peifer, M. 2000. Cadherins in embryonic and neural morphogenesis. Nat. Rev. Mol. Cell Biol. 1:91-100.

112.Edelman, G.M., Gallin, W.J., Delouvee, A., Cunningham, B.A., and Thiery, J.P. 1983. Early epochal maps of two different cell adhesion molecules. Proc. Natl. Acad. Sci. U. S. A. 80:4384-4388.

113. Reichmann, E., et al. 1992. Activation of an inducible c-FosER fusion protein causes loss of epithelial polarity and triggers epithelial-fibroblastoid cell conversion. Cell. 71:1103-1116.

114.Gottardi, C.J., Wong, E., and Gumbiner, B.M. 2001. E-cadherin suppresses cellular transformation by inhibiting beta-catenin signaling in an adhesionindependent manner. J. Cell Biol. 153:1049-1060.

115. Muta, H., et al. 1996. E-cadherin gene mutations in signet ring cell carcinoma of the stomach. Jpn. J. Cancer Res. 87:843-848.

116.Saito, A., et al. 1999. Disruption of E-cadherinmediated cell adhesion systems in gastric cancers in young patients. Jpn. J. Cancer Res. 90:993-999.

117. Hirohashi, S. 1998. Inactivation of the E-cadherinmediated cell adhesion system in human cancers. Am. J. Pathol. 153:333-339.

118.Birchmeier, W., and Behrens, J. 1994. Cadherin expression in carcinomas: role in the formation of cell junctions and the prevention of invasiveness. Biochim. Biophys. Acta. 1198:11-26.

119. Blanco, M.J., et al. 2002. Correlation of Snail expression with histological grade and lymph node status in breast carcinomas. Oncogene. 21:3241-3246.

120. Comijn, J., et al. 2001. The two-handed E box binding zinc finger protein SIP1 downregulates E-cadherin and induces invasion. Mol. Cell. 7:1267-1278.

121.Van de Putte, T., et al. 2003. Mice lacking ZFHX1B, the gene that codes for Smad-interacting protein-1, reveal a role for multiple neural crest cell defects in the etiology of Hirschsprung disease-mental retardation syndrome. Am. J. Hum. Genet. 72:465-470.

122. Yokoyama, K., et al. 2001. Reverse correlation of Ecadherin and snail expression in oral squamous cell carcinoma cells in vitro. Oral Oncol. 37:65-71.

123.Yang, J., et al. 2004. Twist, a master regulator of morphogenesis, plays an essential role in tumor metastasis. Cell. 117:927-939.

124. Radisky, D.C., et al. 2005. Rac1b and reactive oxygen species mediate MMP-3-induced EMT and genomic instability. Nature. 436:123-127.

125.Korpal, M., Lee, E.S., Hu, G., and Kang, Y. 2008. The miR-200 family inhibits epithelial-mesenchymal transition and cancer cell migration by direct targeting of E-cadherin transcriptional repressors ZEB1 and ZEB2. J. Biol. Chem. 283:14910-14914.

126.Park, S.M., Gaur, A.B., Lengyel, E., and Peter, M.E. 2008. The miR-200 family determines the epithelial phenotype of cancer cells by targeting the E-cadherin repressors ZEB1 and ZEB2. Genes Dev. 22:894-907.

127. Gregory, P.A., et al. 2008. The miR-200 family and miR-205 regulate epithelial to mesenchymal transition by targeting ZEB1 and SIP1. Nat. Cell Biol. 10:593-601.

128. Paterson, E.L., et al. 2008. The microRNA-200 family regulates epithelial to mesenchymal transition. Scientific World Journal. 8:901-904.

129. Gregory, P.A., Bracken, C.P., Bert, A.G., and Goodall, G.J. 2008. MicroRNAs as regulators of epithelialmesenchymal transition. Cell Cycle. 7:3112-3118.

130.Zavadil, J., Narasimhan, M., Blumenberg, M., and Schneider, R.J. 2007. Transforming growth factorbeta and microRNA:mRNA regulatory networks in epithelial plasticity. Cells Tissues Organs. 185:157-161. 